\title{
Molecular and Phenotypic Descriptions of Cystodermella cinnabarina from Western Himalaya: A New Genus for Pakistan
}

\author{
Abdul Razaq, ${ }^{1,2}$ Sobia Ilyas, ${ }^{1}$ and Abdul Nasir Khalid ${ }^{1}$ \\ ${ }^{1}$ Department of Botany, University of the Punjab, Lahore 54590, Pakistan \\ ${ }^{2}$ Sustainable Development Study Centre, Government College University, Lahore, Pakistan \\ Correspondence should be addressed to Abdul Razaq; ectomycorrhiza@gmail.com
}

Received 9 October 2013; Accepted 21 November 2013

Academic Editor: Ángel Domínguez

Copyright (C) 2013 Abdul Razaq et al. This is an open access article distributed under the Creative Commons Attribution License, which permits unrestricted use, distribution, and reproduction in any medium, provided the original work is properly cited.

\begin{abstract}
Cystodermella cinnabarina is reported here for the first time from the moist temperate forests of western Himalaya and is the first collection of a Cystodermella from Pakistan. This species is redescribed here using morphological and molecular data. The phylogenetic analysis which is based on internal transcribed spacers (ITS) showed that the Pakistani collection clustered distinctly with similar European sequences in the Cystodermella clade. The Italian and north European sequences of this species clustered in two separate subclades and the Pakistani sequences closely matched the Italian sequences. It is evident that the Pakistani population has a very close evolutionary affinity with the Italian individuals rather than those from northern Europe. The species is distributed in Europe, in North America, and now in the western Himalaya of Asia.
\end{abstract}

\section{Introduction}

Cystodermella is small genus which contains only 12 species [1]. Cystodermella is characterized by having lamellae regularly attached to the stipe, a nonpersistent, scaly annulus, hyaline, smooth, and most importantly inamyloid basidiospores [2, 3]. This genus was segregated from Cystoderma Fayod not only on the basis of morphology and colour reaction (inamyloid basidiospores) but molecular findings also supported phenotypic differences (variation of ITS and LSU markers) [4, 5]. Harmaja [2] described this new genus on the basis of morphoanatomical characters and later many researchers used molecular data to determine the relationship of these two genera [6]. After Moncalvo et al. [5], Saar et al. [3] described Cystoderma and Cystodermella as distinct monophyletic genera on the basis of LSS and ITS barcodes. The genus Cystodermella is widely distributed in Europe, North America, and Asia but Cystodermella cinnabarina, which is a conspecific with Cystoderma cinnabarinum, has not been described before from Asia.

Western Himalaya, the part of Himalaya that lies in Pakistan, is floristically rich with more than 750 plant species of coniferous mixed with deciduous vegetation and more than 300 macrofungi $[7,8]$. These forests are renowned for their species richness of euagarics [7, 9-11]. Recently, Razaq et al. [12, 13] described a few species from this area using internal transcribed spacers of ribosomal DNA and elucidated their phylogeographic relationship with European collections. During a field tour in 2010 , a colorful whitegilled mushroom was collected which was identified as Cystoderma sp. in the field and anatomical and molecular studies showed that it is Cystodermella cinnabarina. This genus had never been reported before from the western Himalaya and C. cinnabarina is the first representative of this genus from Pakistan. The Pakistani collection has been described morphologically and molecularly.

\section{Methods}

Basidiocarps were photographed in the field and morphological characters noted. Sections of lamellae were stained with Congo Red and Melzer's reagent. Measurements were made of 25 basidiospores, 20 basidia, and 20 cystidia from each basidioma under the light microscope equipped with a camera lucida. 


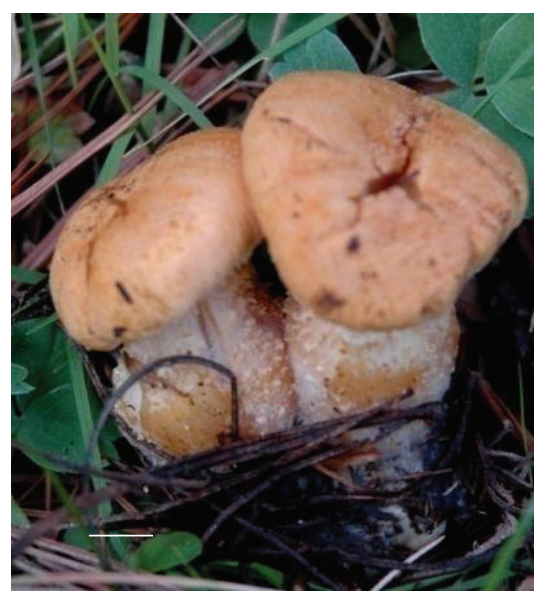

(a)

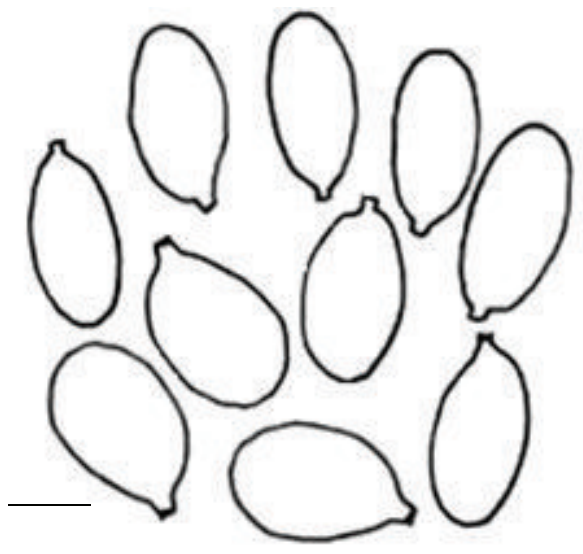

(c)

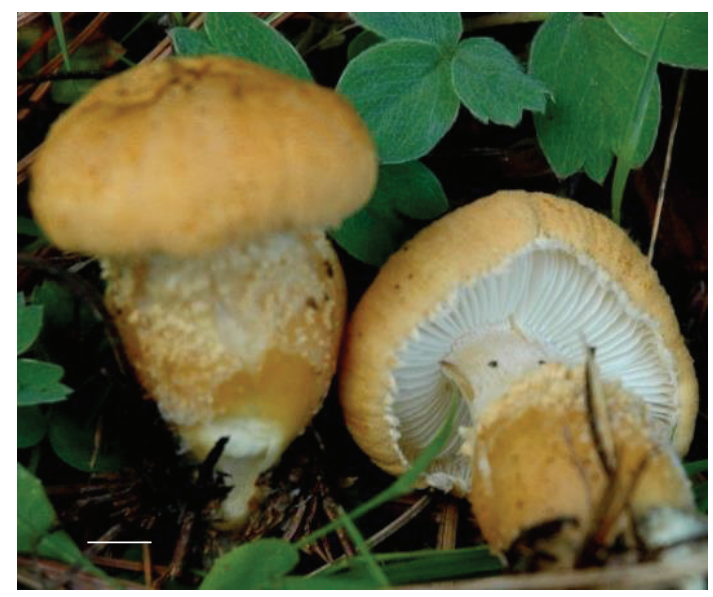

(b)

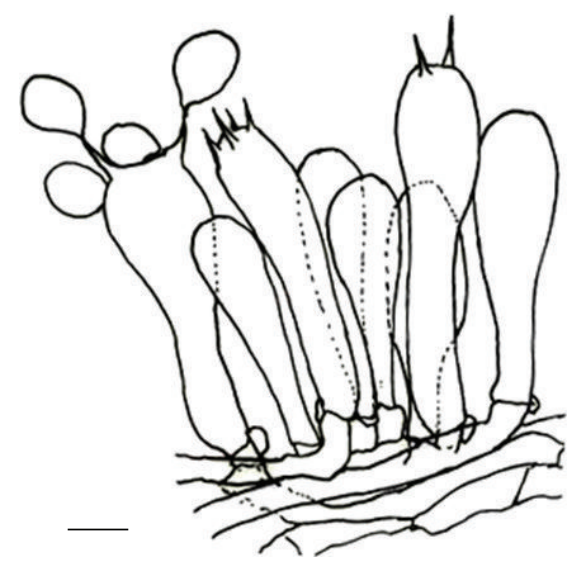

(d)

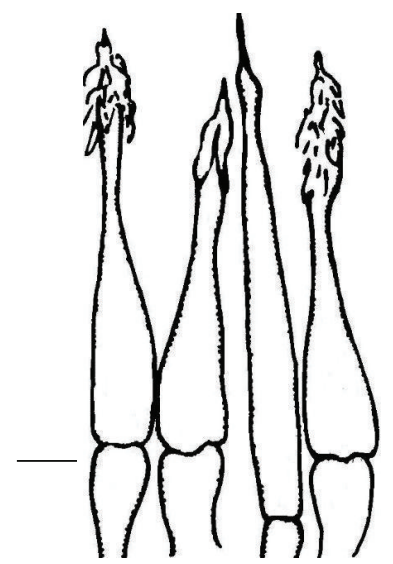

(e)

FIGURE 1: Cystodermella cinnabarina: (a) basidiomata, (b) lamellar side of basidioma, (c) basidiospores, (d) basidia, (e) cheilocystidia bar = (a) $-(\mathrm{b})=1 \mathrm{~cm},(\mathrm{c})=2.5 \mu \mathrm{m},(\mathrm{d})=4.0 \mu \mathrm{m},(\mathrm{e})=8 \mu \mathrm{m}$.

2.1. Molecular Characterization and Phylogenetic Analysis. A sample from a dried specimen of each species was ground in liquid nitrogen and placed in $2 \%$ CTAB buffer and DNA was extracted using the method of Gardes and Bruns [14]. The ITS region of rDNA was amplified using universal primer pair ITS1F and ITS4 [15]. PCR was performed in a $20 \mu \mathrm{L}$ reaction volume following the protocol given by Gardes and Bruns [14]. The size of the PCR product was determined on $1.5 \%$ agarose gel in a gel doc system using the default setting. PCR product was directly sequenced in both directions using the same pair of amplification primers (Macrogen, Korea).

The generated sequence was edited manually using the BioEdit sequence alignment editor version 7.0.9.0 [16]. For initial comparison and alignment of the sequence, Basic Local Alignment Search Tool (BLAST) analysis was performed using the National Center for Biotechnology Information (NCBI), USA database. For further phylogenetic analysis and alignment of the sequence, closely related sequences were retrieved from GenBank. The newly generated sequence and the retrieved sequences were aligned using ClustalW program of Molecular Evolutionary Genetics Analysis (MEGA. 5.1) software [17]. This same software was used for phylogenetic analysis. The maximum likelihood (ML) method was based on the Jukes-Cantor model of nrITS sequences using Nearest-Neighbor-Interchange (NNI) as the ML heuristic search method. All gaps were treated as data and the phylogeny was tested by a bootstrap value of 1000 replicates. Consensus nucleotide sequences of material examined were deposited in the European Molecular Biology Laboratory (EMBL) database.

\section{Results}

\subsection{Taxonomy}

Cystodermella Cinnabarina. (Alb. \& Schwein.) Harmaja [2], Figures 1(a)-1(e).

Morphoanatomical Characterization. Pileus $2.5-6 \mathrm{~cm}$ across, hemispherical to broadly convex, orange-brown to brownish red, fleshy, surface uniform with granular to fine warts over the whole surface, centrally depressed, margins white, involute, undulating, and striated. Flesh white, soft, and up to $0.5 \mathrm{~cm}$ thick at disc. Lamellae off white to cream, adnexed, crowded, and edges entire; partial veil orange-brown, entirely covering the gills in premature stages, and drooping when 
mature. Stipe $2.0-5 \times 0.5-1 \mathrm{~cm}$, hollow, swollen, dry, white scales in the form of sheath covering half of the stipe, white above the veil, and dark orange-brown on lower side.

Basidiospores $4.0-6.0 \times 2.5-4.5 \mu \mathrm{m}$, broadly ellipsoid, smooth, hyaline, with light green contents in $5 \% \mathrm{KOH}$, thin-walled, and inamyloid in Melzer's reagent. Basidia $21.0-68.5 \times 5.5-7.5 \mu \mathrm{m}, 4$-spored, clavate to club shaped, smooth, thin-walled, and hyaline to pale yellow in 5\% $\mathrm{KOH}$ with clamps at base. Cheilocystidia and pleurocystidia, $47.0-59.5 \times 5.5-6.5 \mu \mathrm{m}$, spear-shaped, hyaline, clustered, thin-walled, ventricose below, tapering to a long, narrow neck, apex covered with crystals. Trama consists of loosely arranged hyphae $4-6 \mu \mathrm{m}$.

3.2. Material Examined: Pakistan. Gilgit-Baltistan, Fairy Meadows $\left(34.400570^{\circ} \mathrm{S} 150.891738^{\circ} \mathrm{E}\right)$, in a pine forest of the Himalayan wet temperate region, at $3306 \mathrm{~m}$ a.s.l., July 21, 2010, A Rrazaq, A N Khalid \& S Ilyas, SRS-34 \& SR35 (Herbarium \# LAH 21071034, and 21071035), GenBank accession \# HF559380, and HF559381.

3.3. Habitat and Distribution. In Pakistan this species was collected from a forest which has many decaying logs of coniferous trees and this specimen was collected from litter which is under decaying process. This species is distributed in Asian countries, Europe, America, and Africa [18, 19].

3.4. Molecular Characterization and Phylogenetic Analysis. The target region comprising internal transcribed spacers (ITS1 \& ITS2) and 5.8S of rDNA gave fragments of approximately $700 \mathrm{bp}$ on amplification in the polymerase chain reaction (PCR) using fungal universal primer pairs (ITS1F and ITS4). The initial BLAST analysis of nucleotide sequences revealed that the Pakistani collections (HF559380.1 and HF559381.1) had their maximum match with Cystoderma cinnabarinum (GenBank accession \# JF907975.1), 99\% query coverage, $E$ value 0 . All the sequences in the BLAST analysis of our sequence belong to the same genus or to a different genus in the same family. The percentage base similarity was found to be maximum with Cystoderma cinnabarinum (JF907975.1), an Italian collection submitted from USA. Other closely related BLAST sequences showed 99\%, 99\%, 99\%, and 99\% similarity with C. superbum (GenBank accession JF907974.1, Italy), C. terrei (GenBank accession \# JF907973.1, Italy), Cystodermella cinnabarina (GenBank accession \# AM946515.1, Russia-Northern Caucasus), and C. cinnabarina (GenBank accession \# AM946514.1, Estonia), respectively.

The phylogenetic analysis of the Cystodermella species collected from Pakistan was carried out using the maximum likelihood method. The sequences included in this analysis had initially 1332 characters, from which 701 characters were used in the final analysis after trimming the alignment from both $5^{\prime}$ and $3^{\prime}$ sides. In this phylogenetic analysis, the gaps are treated as data. A total of 31 sequences were included in this analysis, which clustered into two major clades: the Cystodermella clade and the Cystoderma Fayod clade (Figure 2). One species of Cystodermella cinnabarina which had been collected from the Himalayan wet temperate forests of Pakistan was close to the sequences of Cystodermella cinnabarina (AM946513.1, AM946512.1, AM946514.1, and AM946515.1) and Cystoderma cinnabarinum (JF907975.1) with a significant bootstrap value. The presence of cheilocystidia and inamyloid spores identifies the species as Cystodermella cinnabarina. Both Cystoderma cinnabarinum and C. terrei are synonyms as they both fall in same clade (Figure 2).

\section{Discussion}

Cystodermella is a newly erected genus whose generic characters are mostly based on molecular studies [5]. Harmaja [2] created this genus from already existing species of Cystoderma; therefore, species of both genera are closely related morphologically but clustered monophyletically in distinct clades when analyzed on a molecular basis [3]. Cystodermella cinnabarina is characterized by the orangish brown pileus, nettle hair, or spear shaped cystidia with encrusted apices and inamyloid basidiospores (a generic character). Pileus coloration of Cystodermella cinnabarina and Cystoderma terrei and C. cinnabarinum is almost similar $[18,19]$ and Saar et al. [3] proved the conspecific nature of C. cinnabarinum, in molecular analysis, with Cystodermella cinnabarina.

Morphologically, the Pakistani collection is in close agreement with the description of $C$. cinnabarina given by Saar [19] with the exception of a somewhat larger spore size (Pakistani collection 4.0-6.0 × 2.5-4.5 $\mu \mathrm{m}$ versus European collection $3.5-5.0 \times 2.0-2.5 \mu \mathrm{m})$. The spore size of our collection is very close to Cystoderma carcharias (Pers.) Fayod that is reported from India but that species differs in its white pileus and amyloid basidiospores [20]. Spore size differences in C. cinnabarina can be correlated with continental drift and geographical isolation of both populations and variation in their genetic material which is a useful tool for adapting to different environments. This genetic relatedness and these differences are also observed in phylogenetic analysis, in this study, of different populations of this species from different areas of the world.

In the phylogenetic analysis, Cystoderma and Cystodermella distinctly clustered in two clades (Figure 2). Cystodermella clade is comprised of two subclades, C. cinnabarina and the rest of the species of Cystodermella; the former subclade has two sequences of Cystoderma cinnabarinum and C. terrei, the synonyms, and one sequence of C. superbum. Three sequences of Cystoderma present in the Cystodermella clade represent Cystodermella cinnabarina. Among these sequences, Cystoderma superbum (JF907974) is misidentified as it clustered clearly with sequences of Cystodermella cinnabarina while the two published vouchered collections of C. superbum (AM946503 and AM946504) clustered independently in the Cystoderma clade (Figure 2). The Asian collection from Pakistan clustered with Italian sequences indicating that the Pakistani population, genetically, is more similar to the Italian collection rather than those from Sweden, Estonia, and Russia. The last three populations are genetically more cohesive and found towards the Arctic Circle or in an isotherm area of prolonged cold climate while 


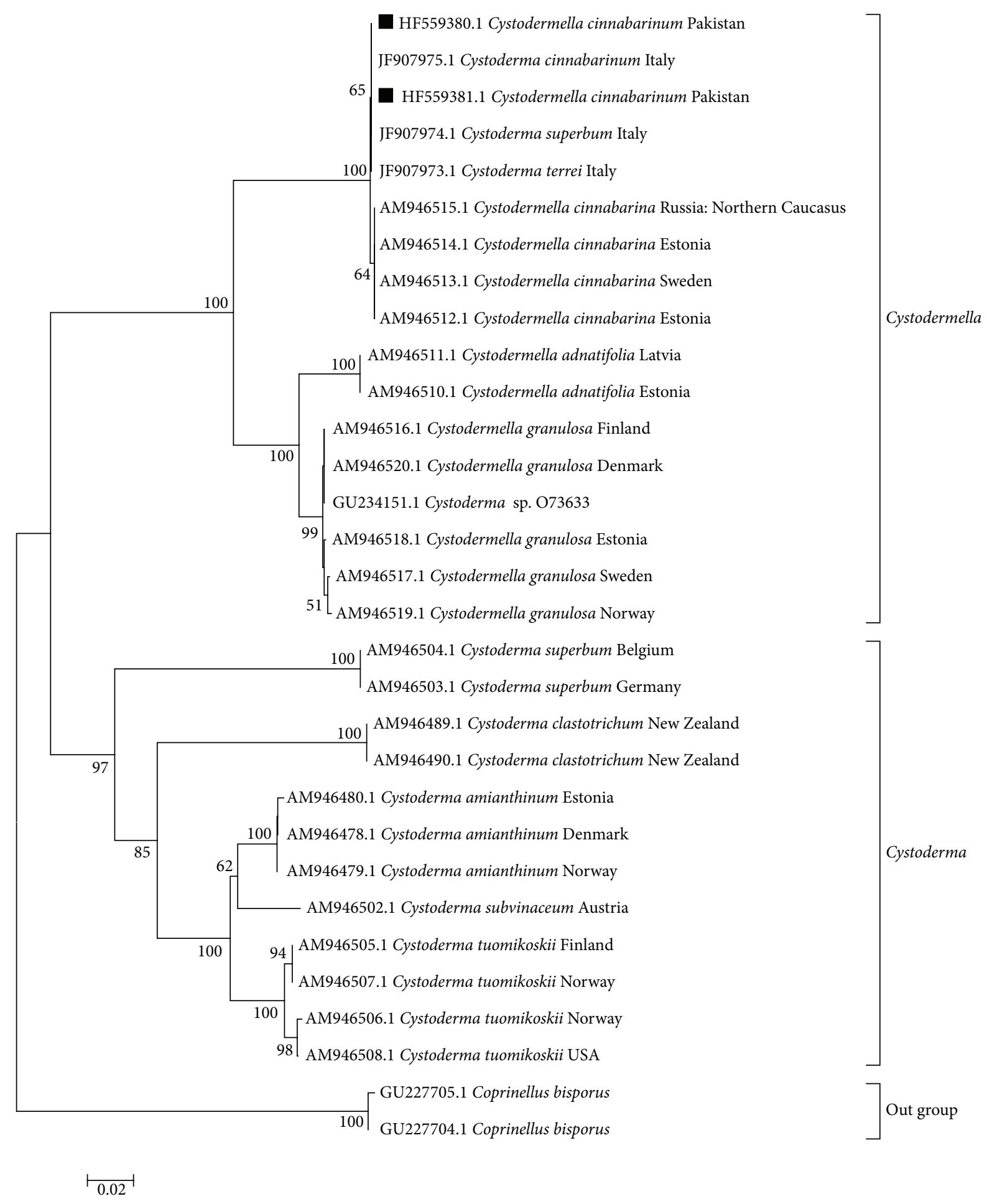

Figure 2: Phylogenetic analysis of Cystodermella cinnabarina has been inferred using maximum likelihood method. The percentage of replicate trees in which the associated taxa clustered together in the bootstrap test (1000 replicates) is shown next to the branches and those below $50 \%$ are collapsed. The analysis involved 31 nucleotide sequences. All positions containing gaps and missing data were eliminated. There were a total of 449 positions in the final dataset. 
the first two populations experience a temperate climate and are close to the equator. Geml et al. 2012 [21] also described this type of biogeographic and allopathic pattern among different European ectomycorrhizal fungi and also explained their phylogeographic origins.

Cystodermella was believed to be well distributed in the world and the greatest diversity is known from north temperate and boreal zones [2]. Osmundson et al. [22] reported Cystoderma cinnabarinum (JF907974) from Italy while Sesli [23] described its presence from Turkey. This species is also common in Estonia, Russia (Northern Caucasus), Sweden [3], North America, and Africa [19] but from the subcontinental plate or Indian plate, it is reported for the first time.

\section{Acknowledgments}

This work was financed by the Higher Education Commission of Pakistan under Indigenous 5000 Fellowship and International Research Support Initiative Program (IRSIP). Authors really thank Dr. Genevieve Gates and Dr. David Ratkowsky from UTAS for correcting and improving the paper.

\section{References}

[1] P. M. Kirk, P. F. Cannon, D. W. Minter, and J. A. Stalpers, Ainsworth \& Bisby's Dictionary of the Fungi, CAB International, Wallingford, UK, 10th edition, 2008.

[2] H. Harmaja, Amylolepiota, Clavicybe and Cystodermella, New Genera of the Agaricales, Karstenia, 2002.

[3] I. Saar, K. Põldmaa, and U. Kõljalg, "The phylogeny and taxonomy of genera," Mycological Progress, vol. 8, no. 1, pp. 5973, 2009.

[4] I. Saar and B. Kullman, "Nuclear DNA content and spore dimensions in some species of the genus Cystoderma," Folia Cryptogam Est, vol. 36, pp. 87-94, 2000.

[5] J.-M. Moncalvo, R. Vilgalys, S. A. Redhead et al., "One hundred and seventeen clades of euagarics," Molecular Phylogenetics and Evolution, vol. 23, no. 3, pp. 357-400, 2002.

[6] M. Capelari and T. Asai, Cystoderma, Cystodermella and Ripartitella in Atlantic Forest, São Paulo State, Brazil, 2009.

[7] S. Ahmad, S. H. Iqbal, and A. N. Khalid, Fungi of Pakistan, Sultan Ahmad Myco. Soc. Pak. Nabiza printers, Lahore, Pakistan, 1997.

[8] I. Samina and K. Salman, "Impacts of protection on floral diversity of himalayan moist temperate forests of galyat," Pakistan Journal of Environment, vol. 01, no. 04, pp. 119-125, 2012.

[9] A. R. Niazi, S. H. Iqbal, and A. N. Khalid, "Biodiversity of Mushrooms and ectomycorrhizas. 1. Russula brevipes Peck., and its ectomycorrhiza-A new record from Himalayan moist temperate forests of Pakistan," Pakistan Journal of Botany, vol. 38 , no. 4, pp. 1271-1277, 2006.

[10] A. Razaq, A. N. Khalid, and E. C. Vellinga, "Lepiota himalayensis sp. nov. (Basidiomycota, Agaricales), a new species from Pakistan," Mycotaxon, vol. 121, pp. 319-325.

[11] A. Razaq, A. N. Khalid, and S. Ilyas, "Tricholomopsis flammula Métrod ex Holec (Basidiomycota, Agaricales)-an addition to the mushroom flora of Pakistan based on molecular identification," Pakistan Journal of Botany, vol. 44, pp. 413-416, 2012.
[12] A. Razaq, A. N. Khalid, and S. Ilyas, "Molecular identification of Lepiota acutesquamosa and L. cristata (Basidiomycota, Agaricales) based on ITS-rDNA barcoding from Himalayan moist temperate forests of Pakistan," International Journal of Agriculture and Biology, vol. 15, pp. 315-318, 2013.

[13] A. Razaq, A. N. Khalid, and S. Ilyas, "Molecular identification of Marasmius oreades - an edible mushroom from Pakistan based on ITS-rDNA data," Pakistan Journal of Agricultural Sciences, vol. 50, no. 3, pp. 415-419, 2013.

[14] M. Gardes and T. D. Bruns, "ITS primers with enhanced specificity for basidiomycetes-application to the identification of mycorrhizae and rusts," Molecular Ecology, vol. 2, no. 2, pp. 113-118, 1993.

[15] T. J. White, T. Burns, S. Lee, and J. Taylor, "Amplification and direct sequencing of fungal ribosomal RNA genes for phylogenetics," in PCR Protocols, A Guide To Methods and Applications, M. A. Innis, D. H. Gelfand, J. J. Sninsky, and T. J. White, Eds., Academic Press, San Diego, Calif, USA, 1990.

[16] T. A. Hall, Bioedit Version 7.0.4.Department of Microbiology, North Carolina State University, 2005.

[17] K. Tamura, D. Peterson, N. Peterson, G. Stecher, M. Nei, and S. Kumar, "MEGA5: molecular evolutionary genetics analysis using maximum likelihood, evolutionary distance, and maximum parsimony methods," Molecular Biology and Evolution, vol. 28, no. 10, pp. 2731-2739, 2011.

[18] J. Breitenbach and F. Kränzlin, Fungi of Switzerland, vol. 4, Mykologia, Luzern, Switzerland, 2nd edition, 1995.

[19] I. Saar, "The genera Cystoderma and Cystodermella (Tricholomataceae) in temperate eurasia," Mycotaxon, vol. 86, pp. 455473, 2003.

[20] Jamaluddin, M. G. Goswamy, and B. M. Ojha, Fungi of India 1989-2001, Scientific Publisher, Jodhpur, India, 2004.

[21] J. Geml, I. Timling, C. H. Robinson et al., "An arctic community of symbiotic fungi assembled by long-distance dispersers: phylogenetic diversity of ectomycorrhizal basidiomycetes in Svalbard based on soil and sporocarp DNA," Journal of Biogeography, vol. 39, no. 1, pp. 74-88, 2012.

[22] T. W. Osmundson, V. A. Robert, C. L. Schoch, L. J. Baker, A. Smith et al., "Filling gaps in biodiversity knowledge for macrofungi: contributions and assessment of an herbarium collection DNA barcode sequencing project," PLoS ONE, vol. 8, no. 4, 2013.

[23] E. Sesli, “Cystoderma cinnabarinum (Alb. \& Schwein.) Fayod, a new Turkish mycota record," Turkish Journal of Botany, vol. 29, no. 6, pp. 463-466, 2005. 

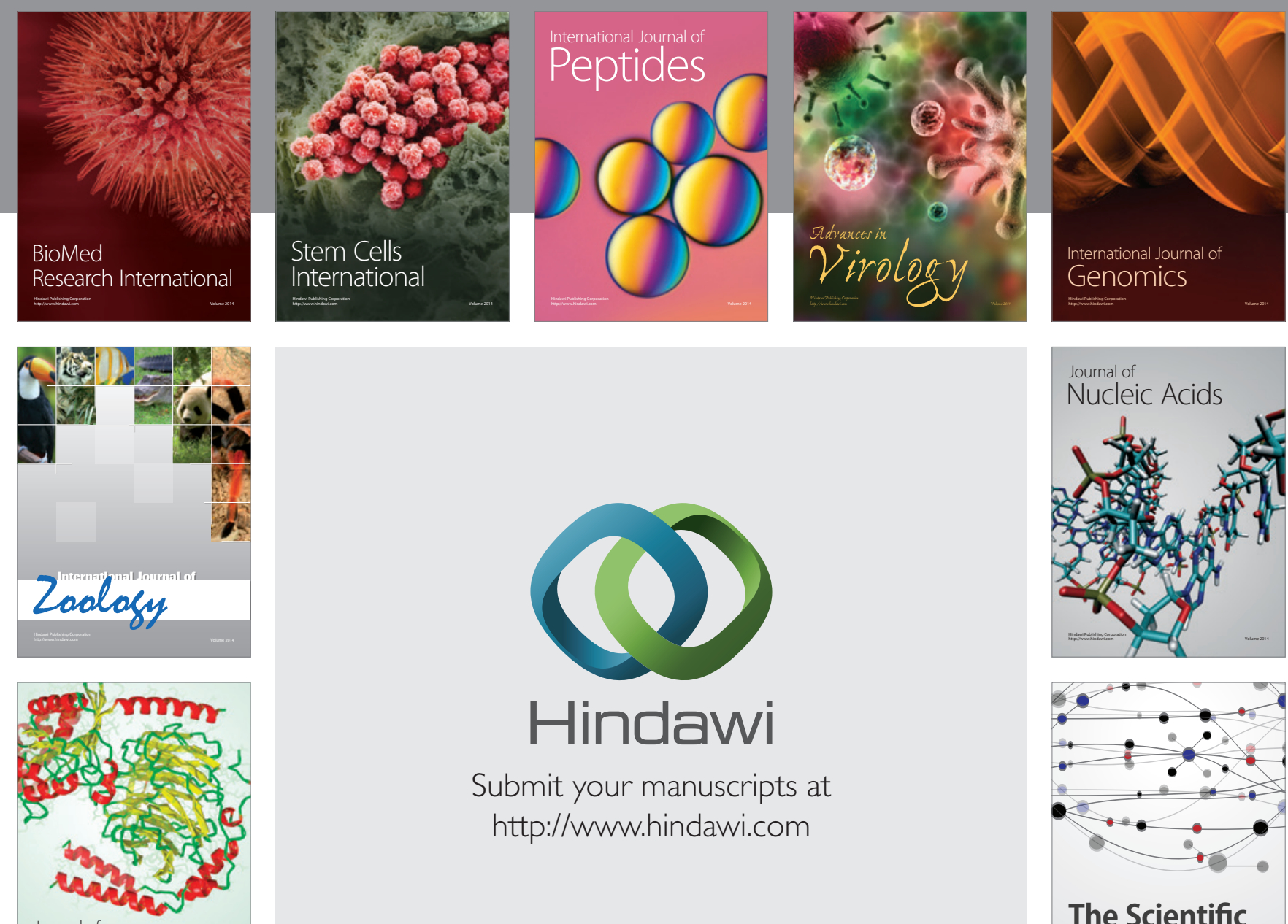

Submit your manuscripts at

http://www.hindawi.com

Journal of
Signal Transduction
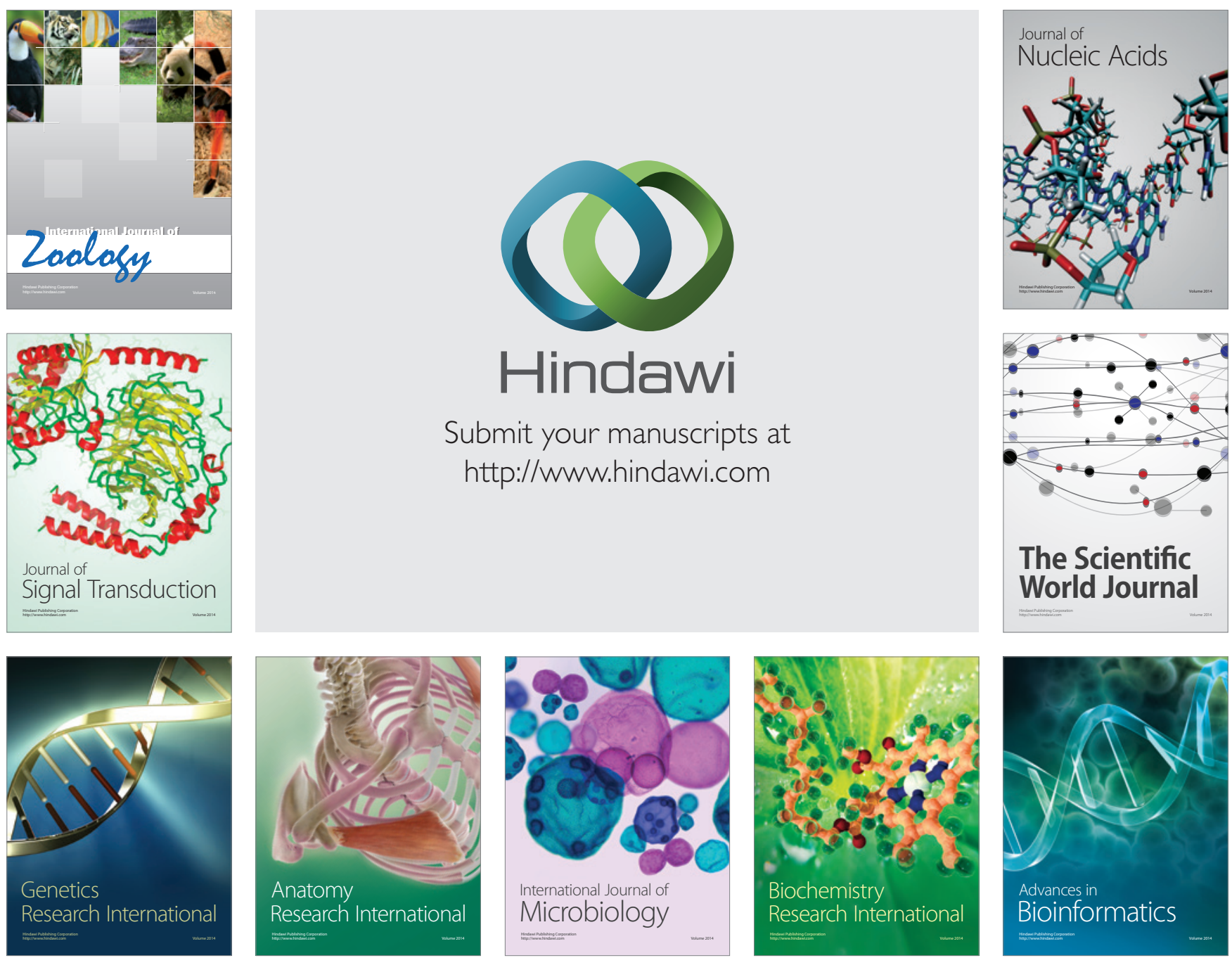

The Scientific World Journal
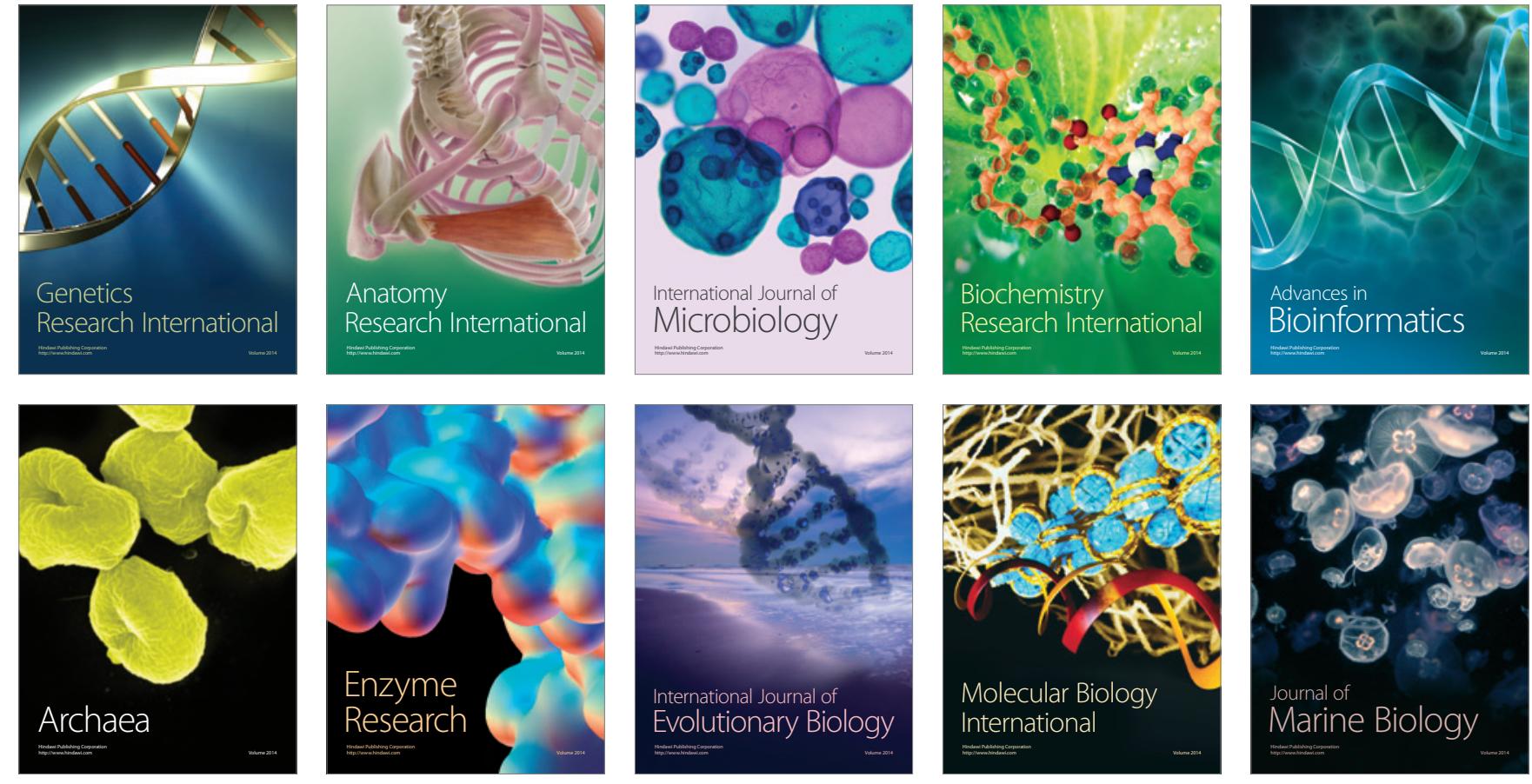